\title{
PERFORMANCE OF AGED LAYING FAYOUMI HENS FED PYRIDOXINE OR FOLIC ACID-SUPPLEMENTED DIETS
}

\author{
Mohamed, S. Bahnas \\ Fac. of Agric., Poultry Production Dept., Fayoum Univ., Fayoum, Egypt. \\ ABSTRACT:
}

A total number of 144 hens and 12 cocks of 48 wk old from Fayoumi strain were used to determine whether hatchability can be improved by using folic acid or pyridoxine supplementation in diets during the late stages of egg production. Birds were divided into 3 equal groups (48 hens +4 cocks each) of 12 replicates each. A corn-soybean meal basal experimental layer diet was formulated of being $16.5 \% \mathrm{CP}$ and 2700 kcal.ME $/ \mathrm{kg}$ diet. Three dietary treatments were used: unsupplemented corn-soybean meal basal diet; the basal diet supplemented with $1 \mathrm{mg}$ folic acid/kg; and the basal diet supplemented with $6 \mathrm{mg}$ pyridoxine $/ \mathrm{kg}$. Response criteria included egg production, egg fertility and hatchability as well as egg quality traits. Weekly data responses were averaged across 4-wk periods and recorded in three grouped time periods over the production periods. The following results were obtained: 1) Feeding pyridoxine-supplemented diet caused the highest egg weight; however, feeding folic acid or pyridoxinesupplemented diets resulted in significantly higher egg number and egg mass and better feed conversion ratio as compared to feeding control diet. 2) Feeding folic acid-supplemented diet caused the highest hatchability $\%$ and salable chicks \%; however, pyridoxine-supplemented diets caused the highest chick weight. Moreover, feeding folic acid or pyridoxinesupplemented diets resulted in significantly higher fertility $\%$ as compared to feeding control diet. 3) Feeding folic acid-supplemented diet caused the highest egg shape index; however, feeding folic acid or pyridoxinesupplemented diets resulted in significantly higher yolk $\%$, shell $\%$, shell thickness and yolk height as compared to feeding control diet. On the other hand, albumin \% and albumin height were not significantly affected by different treatments. From the nutritional point of view, under the present experimental conditions, it is advisable to add $1 \mathrm{mg}$ folic acid or 6 $\mathrm{mg}$ pyridoxine $/ \mathrm{kg}$ of aged Fayoumi laying hen diet. Such practice helps in improving laying performance, fertility, hatchability and egg quality and seems to be advantageous in decreasing chick abnormalities.

Key words: Egg production, hatchability, hatching parameters, egg quality, Fayoumi hens, pyridoxine, folic acid.

\section{INTRODUCTION}

Egg production depends on many characters. Age has a negative effect on productive and reproductive performance of layer hens especially at the end of production cycle. Seker et al. (2004) reported that the parental age had significant effect on the fertility and hatchability of Japanese quail fertile eggs, but it had no significant effect on both dead eggs and one-day old chick weight. Egg fertility and hatchability have depended upon different items and mainly by flock age

Fayoum J. Agric. Res. \& Dev., Vol.23, No.1, January, 2009 
(Tona et al., 2001). The older ages of the flock have led an increase in eggs and a reduction in eggshell and accordingly a decrease in hatchability of incubated eggs (Elibol $\boldsymbol{e t}$ al., 2002). Therefore, the eggs obtained from very old flocks are not considered as appropriate for the hatching activities (Sahan and Ipek 2000).

Egg production is a result of nutritional status that acts to allow the chicken to express fully its genetic potential. Vitamins are involved in most metabolic processes and are an integral part of embryonic development, therefore suboptimal levels of these nutrients in diets are known to result in negative responses to both parent and offspring performance.

Pyridoxine is one of the three major chemical forms of vitamin $\mathrm{B}_{6}$ that is a water-soluble vitamin (Bender, 1989). It performs a wide variety of functions in body and is essential for good health. It is a coenzyme involved in amino acid metabolism. It is also essential for red blood cell metabolism. The nervous and immune systems need vitamin $B_{6}$ to function efficiently (Trakatellis $\boldsymbol{e t ~ a l . , ~ 1 9 9 7 ) . ~}$ It is also needed for the conversion of tryptophan to niacin (Shibata $\boldsymbol{e t}$ al., 1995). Vitamin $\mathrm{B}_{6}$ is needed to make hemoglobin that carries oxygen to tissues. A vitamin $\mathrm{B}_{6}$ deficiency can result in a form of anemia (Leklem, 1999) that is similar to iron deficiency anemia. Vitamin $\mathrm{B}_{6}$, through its involvement in protein metabolism and cellular growth, is important to the immune system. It helps maintain the health of lymphoid organs (thymus, spleen, and lymph nodes) that make white blood cells. Animal studies show that a vitamin $\mathrm{B}_{6}$ deficiency can decrease antibody production and suppress your immune response (Chandra and Sudhakaran, 1990). Vitamin $B_{6}$ also helps maintain blood glucose within a normal range. When caloric intake is low, body needs vitamin $\mathrm{B}_{6}$ to help convert stored carbohydrate or other nutrients to glucose to maintain normal blood sugar levels.

Folic acid status is linked to increased serum levels of the sulfur amino acid homocystine, due to the role of folic acid that plays as co-factor in the methylation of homocystine to form methionine (House et al., 1999). Increasing the folic acid content of eggs make the egg as an important source of dietary folic acid lead to consumer acceptance of this commodity as a healthful product (House et al., 2002).

Fayoumi chicken is well known and wide distributed in Upper Egypt and its characteristics of egg production and egg quality are still low. Therefore, the present work was carried out to study the effect of supplementing pyridoxine and folic acid into diet on productive performance, egg quality and egg components of Fayoumi hens during their late stage of production.

\section{MATERIALS AND METHODS}

Experimental Birds and Housing

A total number of 144 hens and 12 cocks from Fayoumi chickens of 48 wk old were fasted overnight, individually leg-banded, weighed to the nearest $1 \mathrm{~g}$ and randomly divided into 3 equal groups ( 48 hens +4 cocks each) of 12 replicates each. The average initial live body weight (BW) was insignificantly different in all groups. The groups were randomly re-housed in 12 suitable floor laying pens $(2 \mathrm{~m}$ length $\times 2 \mathrm{~m}$ width) with chopped wheat straw litter and supplied with one automatic drinker and one manual feeder.

Fayoum J. Agric. Res. \& Dev., Vol.23, No.1, January, 2009 
PERFORMANCE OF AGED LAYING FAYOUMI HENS FED.............. 3

\section{Experimental Diets and Treatments:}

A corn-soybean meal basal experimental layer diet was formulated of being $16.5 \% \mathrm{CP}$ and $2700 \mathrm{kcal} . \mathrm{ME} / \mathrm{kg}$. The composition and chemical analysis of the experimental diets is presented in Table (1). The first diet was considered as a control diet and contained the NRC (1994) recommended level of folic acid and pyridoxine. The second treatment group contained $1 \mathrm{mg}$ folic acid/kg diet. The third ones contained $6 \mathrm{mg}$ pyridoxine $/ \mathrm{kg}$ diet.

\section{Management:}

Birds in all experimental treatments were kept under similar managerial and hygienic conditions. The experimental layer diet were weekly mixed and offered, in mash form. Also, fresh clean water was freely available and artificial light was provided 16 hours per day during the experimental period.

\section{Measurements and Methods of Interpreting Results}

The following criteria were measured and/or calculated in the present study

\section{Laying Performance:}

Egg number (EN), egg weight (EW), egg mass (EM), feed intake (FI) and feed conversion ratio $(\mathrm{FCR})(\mathrm{kg}$ feed $/ \mathrm{kg}$ egg) were recorded for each hen for each 4weeks period from 48 to 60 weeks of age.

\section{Egg Quality Traits:}

Percentages of yolk, albumen and shell were calculated. Egg shape index (ESI) was estimated as the ratio of the egg maximum width to its length. Shell thickness was estimated on the membraneless shell by using Ames shell thickness gauge to the nearest $\mu \mathrm{m}$. Thick albumen height $(\mathrm{AH})$ and yolk height ( $\mathrm{YH})$ were measured to the nearest mm.with a tripod micrometer

\section{Hatching Parameters:}

At 48-52, 54-56 and 56-60 weeks of age, eggs were collected. At each age, egg collection continued until a total number of 90 eggs per each treatment had been collected after removing visibly dirty, misshapen or cracked eggs. Eggs for each treatment were placed into 3 trays (30 eggs per tray). Thereafter, eggs were fumigated for $20 \mathrm{~min}$. with formaldehyde gas. The experiment was conducted using electronically controlled single-stage incubators (Petersime, Model 576). The temperature and humidity recommended by the incubator manufacturer were maintained. Fertility was estimated by the number of fertile eggs at the $8^{\text {th }}$ days of the incubation in percent of total number of eggs set. Hatchability was estimated by the number of healthy chicks hatched in percent of total number of fertile eggs set.

\section{Proximate and Statistical Analysis:}

Chemical analysis of representative samples of the experimental diets was carried out in triplicate according to the standard methods outlined by A.O.A.C. (1980). Data obtained were statistically analyzed by a one way analysis of variance according to Steel and Torrie (1980) including analysis of variance. Also, SPSS computer statistical program for MS Windows release 6.0 June 1993 was used. Differences among means were tested using Duncan's Multiple Range Test (Duncan, 1955) with a 5\% level of probability.

Fayoum J. Agric. Res. \& Dev., Vol.23, No.1, January, 2009 
Mohamed, S. Bahnas

Table 1: Composition and calculated analysis of the experimental layer basal diet.

\begin{tabular}{|l|c|}
\hline \multicolumn{1}{|c|}{ Ingrediens } & Percentage \\
\hline Yellow Corn, ground & 62.84 \\
\hline Soybean meal(44\% CP) & 26.04 \\
\hline Wheat bran & 1.12 \\
\hline Dicalcium phosphate & 1.54 \\
\hline Limestone & 7.77 \\
\hline Common salt $(\mathrm{NaCl})$ & 0.33 \\
\hline Premix* & 0.30 \\
\hline DL-Methionine & 0.06 \\
\hline Total & 100.00 \\
\hline Calculated values $(\%)$ & \\
\hline CP \% & 16.50 \\
\hline ME (kcal/kg) & 2700 \\
\hline CF \% & 3.46 \\
\hline EE \% & 2.82 \\
\hline Ca \% & 3.33 \\
\hline Av. Phosphorus \% & 0.41 \\
\hline Lysine \% & 0.92 \\
\hline Methionine \% & 0.35 \\
\hline Methionine + Cyst \% & 0.63 \\
\hline Sodium \% & 0.17 \\
\hline The premix (Vit. \& $\%$ ) & 01 \\
\hline
\end{tabular}

*The premix (Vit. \& Min.) was added at a rate of $3 \mathrm{~kg}$ per ton of diet and supplied the following (as mg or I.U) per kg of die): Vit. A 12000 I.U., Vit. D 2000 I.U., Vit. E 40 mg, Vit. $\mathrm{K}_{3} 4 \mathrm{mg}$, Vit. $\mathrm{B}_{1} 3 \mathrm{mg}$, Vit. $\mathrm{B}_{2} 6 \mathrm{mg}$, Vit. $\mathrm{B}_{6} 4 \mathrm{mg}$, Vit. $\mathrm{B}_{12} 0.03 \mathrm{mg}$, Niacin $30 \mathrm{mg}$, Biotin $0.08 \mathrm{mg}$, Pantothenic acid $12 \mathrm{mg}$, Folic acid $1.5 \mathrm{mg}$, Choline chloride $700 \mathrm{mg}$, Mn 80 $\mathrm{mg}$, Cu $10 \mathrm{mg}$, Se $0.2 \mathrm{mg}$, I $40 \mathrm{mg}$, Fe $40 \mathrm{mg}$, Zn $70 \mathrm{mg}$ and Co $0.25 \mathrm{mg}$.

\section{RESULTS AND DISCUSSION}

\section{Laying Performance:}

The results obtained for laying hen performance in terms of egg weight (EW) egg number (EN), egg mass (EM), feed intake (FI) and feed conversion ratio (FCR) at 48-52, 52-56 and 56-60 weeks of age are shown in Table 2.

At all ages, hens receiving pyridoxine-supplemented diet had significantly high EW. On the other hand, hens fed folic acid or pyridoxine-supplemented diet had significantly higher EN and EM and better FCR than those fed control diet.

The improvement in the final EW and EN of hens fed folic acid or pyridoxinesupplemented diets in the current study agrees with those reported by Robel (1993) who mentioned that turkey egg weight was significantly increased with the increase of folic acid supplementation but disagree with the results of House et al., (2002) who found no significant difference due to folic acid supplementation on egg weight or production. Also, Keshavarz (2003) reported that certain manipulations of the combination of folic and other vitamins have the potential effect to reduce egg weight without affecting egg production. Ryu et al., (1995) found a significant linear feed conversion response to dietary folic acid for broiler chicks

Fayoum J. Agric. Res. \& Dev., Vol.23, No.1, January, 2009 
PERFORMANCE OF AGED LAYING FAYOUMI HENS FED............ 5

The non-significant differences in FI between hens fed folic acid or control diets during the tested periods in the current study disagree with the results of House et al., (2002) who stated that feed consumption was slightly depressed at level of 4-16 $\mathrm{mg}$ folic/ $\mathrm{kg}$ intake.

Table 2: Laying performance of hens fed the experimental diets at 48-52, 52$56,56-60$ and $48-60$ weeks of age.

\begin{tabular}{|c|c|c|c|}
\hline \multirow{2}{*}{ Items } & \multicolumn{3}{|c|}{ Treatments } \\
\hline & Control & Folic acid & Pyridoxine \\
\hline & \multicolumn{3}{|c|}{ 48-52 wks. } \\
\hline Egg weight (EW), g & $47.48 \pm 0.26^{\mathrm{c}}$ & $50.50 \pm 0.06^{\mathrm{b}}$ & $51.03 \pm 0.02^{\mathrm{a}}$ \\
\hline Egg number (EN), No./hen/period & $15.40 \pm 0.05^{\mathrm{b}}$ & $15.88 \pm 0.09^{\mathrm{a}}$ & $15.81 \pm 0.01^{\mathrm{a}}$ \\
\hline Egg mass (EM), kg/hen & $0.73 \pm 0.01^{\mathrm{b}}$ & $0.80 \pm 0.01^{\mathrm{a}}$ & $0.81 \pm 0.004^{\mathrm{a}}$ \\
\hline Feed intake (FI), kg/hen & $3.00 \pm 0.02$ & $3.00 \pm 0.02$ & $3.10 \pm 0.02$ \\
\hline feed conversion ratio (FCR), $g$ feed/g egg & $4.11 \pm 0.01^{\mathrm{a}}$ & $3.75 \pm 0.02^{\mathrm{b}}$ & $3.83 \pm 0.02^{\mathrm{b}}$ \\
\hline \multicolumn{4}{|c|}{ 52-56 wks. } \\
\hline Egg weight $(\mathrm{EW}), \mathrm{g}$ & $47.67 \pm 0.86^{\mathrm{c}}$ & $49.75 \pm 0.10^{\mathrm{b}}$ & $50.81 \pm .06^{\mathrm{a}}$ \\
\hline Egg number (EN), No./hen/period & $14.43 \pm 0.08^{\mathrm{b}}$ & $15.35 \pm 0.14^{\mathrm{a}}$ & $15.28 \pm 0.09^{\mathrm{a}}$ \\
\hline Egg mass $(\mathrm{EM}), \mathrm{kg} / \mathrm{hen}$ & $0.69 \pm 0.01^{\mathrm{b}}$ & $0.76 \pm 0.01^{\mathrm{a}}$ & $0.78 \pm 0.01^{\mathrm{a}}$ \\
\hline Feed intake (FI), kg/hen & $2.92 \pm 0.05$ & $2.90 \pm 0.04$ & $2.83 \pm 0.02$ \\
\hline feed conversion ratio (FCR), $\mathrm{g}$ feed/g egg & $4.23 \pm 0.08^{\mathrm{a}}$ & $3.82 \pm 3.82^{\mathrm{b}}$ & $3.63 \pm 0.01^{\mathrm{b}}$ \\
\hline \multicolumn{4}{|c|}{ 56-60 wks. } \\
\hline Egg weight $(\mathrm{EW}), \mathrm{g}$ & $48.09 \pm 0.05^{\mathrm{c}}$ & $49.01 \pm 0.06^{\mathrm{b}}$ & $49.86 \pm 0.08^{\mathrm{a}}$ \\
\hline Egg number (EN), No./hen/ & $13.98 \pm 0.09^{\mathrm{b}}$ & $14.82 \pm 0.08^{\mathrm{a}}$ & $14.79 \pm 0.20^{\mathrm{a}}$ \\
\hline Egg mass (EM), kg/hen & $0.67 \pm 0.004^{c}$ & $0.73 \pm 0.004^{\mathrm{a}}$ & $0.74 \pm 0.01^{\mathrm{a}}$ \\
\hline Feed intake (FI), kg/hen & $2.78 \pm 0.02$ & $2.80 \pm 0.02$ & $2.85 \pm 0.05$ \\
\hline feed conversion ratio (FCR), $\mathrm{g}$ feed/g egg & $4.15 \pm 0.01^{\mathrm{a}}$ & $3.84 \pm 0.02^{\mathrm{b}}$ & $4.00 \pm 0.02^{\mathrm{b}}$ \\
\hline \multicolumn{4}{|c|}{ Overall period } \\
\hline Egg weight $(\mathrm{EW}), \mathrm{g}$ & $47.75 \pm 0.09^{\mathrm{c}}$ & $49.75 \pm 0.12^{\mathrm{b}}$ & $50.57 \pm 0.07^{\mathrm{a}}$ \\
\hline Egg number (EN), No./hen/period & $43.81 \pm 0.11^{\mathrm{b}}$ & $46.05 \pm 0.10^{\mathrm{a}}$ & $45.88 \pm 0.08^{\mathrm{a}}$ \\
\hline Egg mass $(\mathrm{EM}), \mathrm{kg} / \mathrm{hen}$ & $2.09 \pm 0.02^{\mathrm{b}}$ & $2.29 \pm 0.01^{\mathrm{a}}$ & $2.33 \pm 0.03^{\mathrm{a}}$ \\
\hline Feed intake (FI), kg/hen & $8.70 \pm 0.09$ & $8.70 \pm 0.07$ & $8.78 \pm 0.05$ \\
\hline feed conversion ratio (FCR), g feed/g egg & $4.16 \pm 0.03^{\mathrm{a}}$ & $3.80 \pm 0.01^{\mathrm{b}}$ & $3.82 \pm 0.02^{\mathrm{b}}$ \\
\hline
\end{tabular}

$a, b, c$ : means in the same row having different letters are significantly different at $p \leq 0.05$.

\section{Hatching Parameters:}

The results obtained for hatching parameters in terms of the percentages of infertile eggs $\%$, dead eggs $\%$, dead chicks $\%$, abnormal chicks $\%$, chick weight, fertility $\%$ and hatchability $\%$ at 50,54 and 58 weeks of age are presented in Table 3.

At all ages, it was noticed that hens fed folic acid or pyridoxine-supplemented diet had significantly higher fertility $\%$ and lower dead and abnormal chicks $\%$ than those fed control diet. The results also indicated that hens fed folic acidsupplemented diet had significantly higher hatchability $\%$ and salable chicks $\%$ as well as lower chick weight $\%$ than those fed pyridoxine-supplemented diet; however, hens fed control diet gave the lowest values in this respect.

Fayoum J. Agric. Res. \& Dev., Vol.23, No.1, January, 2009 
Mohamed, S. Bahnas

Table 3: Hatching parameters \% for laying hens fed the experimental diets at 50, 54 and 58 weeks of age.

\begin{tabular}{|c|c|c|c|}
\hline \multirow{2}{*}{ Items } & \multicolumn{3}{|c|}{ Treatments } \\
\hline & Control & Folic acid & Pyridoxine \\
\hline & \multicolumn{3}{|c|}{50 wks. } \\
\hline Fertility, \% & $91.94 \pm 0.10^{\mathrm{b}}$ & $93.77 \pm 0.03^{\mathrm{a}}$ & $93.99 \pm 0.13^{\mathrm{a}}$ \\
\hline Hatchability, \% & $82.05 \pm 0.49^{c}$ & $86.11 \pm 0.04^{\mathrm{a}}$ & $84.06 \pm 0.22^{b}$ \\
\hline Dead chicks, \% & $1.80 \pm 0.02^{\mathrm{a}}$ & $1.19 \pm 0.03^{\mathrm{b}}$ & $1.18 \pm 0.02^{\mathrm{b}}$ \\
\hline Abnormal chicks, \% & $1.31 \pm 0.05^{\mathrm{a}}$ & $0.66 \pm 0.03^{b}$ & $0.71 \pm 0.05^{\mathrm{b}}$ \\
\hline Salable chicks, \% & $78.94 \pm 0.3^{\mathrm{c}}$ & $84.26 \pm 0.05^{\mathrm{a}}$ & $82.17 \pm 0.04^{b}$ \\
\hline \multirow[t]{2}{*}{ Chick weight, gm } & $32.18 \pm 0.09^{\mathrm{c}}$ & $32.88 \pm 0.07^{\mathrm{b}}$ & $33.54 \pm 0.12^{\mathrm{a}}$ \\
\hline & \multicolumn{3}{|c|}{54 wks. } \\
\hline Fertility, \% & $92.00 \pm 0.10^{\mathrm{b}}$ & $93.27 \pm 0.05^{\mathrm{a}}$ & $93.41 \pm 0.05^{\mathrm{a}}$ \\
\hline Hatchability, \% & $81.04 \pm 0.04^{\mathrm{c}}$ & $85.46 \pm 0.18^{\mathrm{a}}$ & $83.91 \pm 0.31^{\mathrm{b}}$ \\
\hline Dead chicks, \% & $1.91 \pm 0.03^{\mathrm{a}}$ & $1.11 \pm 0.03^{\mathrm{b}}$ & $1.19 \pm 0.03^{\mathrm{b}}$ \\
\hline Abnormal chicks, \% & $1.40 \pm 0.17^{\mathrm{a}}$ & $0.85 \pm 0.04^{\mathrm{b}}$ & $0.92 \pm 0.04^{\mathrm{b}}$ \\
\hline Salable chicks, \% & $77.73 \pm 0.06^{\mathrm{c}}$ & $83.50 \pm 0.04^{\mathrm{a}}$ & $81.80 \pm 0.05^{\mathrm{b}}$ \\
\hline \multirow[t]{2}{*}{ Chick weight, gm } & $32.01 \pm 0.14^{\mathrm{b}}$ & $32.20 \pm 0.26^{\mathrm{b}}$ & $33.21 \pm 0.25^{\mathrm{a}}$ \\
\hline & \multicolumn{3}{|c|}{58 wks. } \\
\hline Fertility, \% & $92.04 \pm 0.01^{\mathrm{b}}$ & $93.10 \pm 0.12^{\mathrm{a}}$ & $93.27 \pm 0.08^{\mathrm{a}}$ \\
\hline Hatchability, \% & $80.21 \pm 0.05^{\mathrm{c}}$ & $84.58 \pm 0.09^{\mathrm{a}}$ & $82.07 \pm 0.07^{\mathrm{b}}$ \\
\hline Dead chicks, \% & $2.04 \pm 0.01^{\mathrm{a}}$ & $1.14 \pm 0.03^{\mathrm{b}}$ & $1.21 \pm 0.02^{\mathrm{b}}$ \\
\hline Abnormal chicks, \% & $1.70 \pm 0.05^{\mathrm{a}}$ & $0.96 \pm 0.02^{b}$ & $1.00 \pm 0.07^{\mathrm{b}}$ \\
\hline Salable chicks, \% & $76.47 \pm 0.02^{\mathrm{c}}$ & $82.48 \pm 0.04^{\mathrm{a}}$ & $79.86 \pm 0.03^{b}$ \\
\hline \multirow[t]{2}{*}{ Chick weight, gm } & $32.02 \pm 0.12^{\mathrm{c}}$ & $32.46 \pm 0.19^{b}$ & $33.06 \pm 0.13^{\mathrm{a}}$ \\
\hline & \multicolumn{3}{|c|}{ Overall mean } \\
\hline Fertility, \% & $91.99 \pm 0.03^{\mathrm{b}}$ & $93.38 \pm 0.01^{\mathrm{a}}$ & $93.56 \pm 0.03^{\mathrm{a}}$ \\
\hline Hatchability, \% & $81.10 \pm 0.10^{c}$ & $85.38 \pm 0.09^{\mathrm{a}}$ & $83.35 \pm 0.10^{\mathrm{b}}$ \\
\hline Dead chicks, \% & $1.92 \pm 0.02^{\mathrm{a}}$ & $1.15 \pm 0.02^{b}$ & $1.19 \pm 0.03^{\mathrm{b}}$ \\
\hline Abnormal chicks, \% & $1.47 \pm 0.03^{\mathrm{a}}$ & $0.82 \pm 0.02^{\mathrm{b}}$ & $0.88 \pm 0.02^{\mathrm{b}}$ \\
\hline Salable chicks, \% & $77.71 \pm 0.07^{\mathrm{c}}$ & $83.41 \pm 0.04^{\mathrm{a}}$ & $81.28 \pm 0.06^{\mathrm{b}}$ \\
\hline Chick weight, gm & $32.07 \pm 0.03^{\mathrm{c}}$ & $32.51 \pm 0.03^{\mathrm{b}}$ & $33.27 \pm 0.02^{\mathrm{a}}$ \\
\hline
\end{tabular}

$\mathrm{a}, \mathrm{b}, \mathrm{c}$ : means in the same row having different letters are significantly different at $\mathrm{p} \leq 0.05$

The increased of one-day old chick weight observed in the present study may be due to the increase in egg weight while is in agreement with these of Kucukyilmaz et al. (2001) who reported that there was a high relationship between the egg weight and the one-day old chick weight of Japanese quail in various studies The improvement observed in fertility and hatchability of eggs produced from hens receiving folic acid or pyridoxine-supplemented diets as compared to those fed control diet in this study could be associated with the improvement in egg shell quality due to feeding folic acid or pyridoxinesupplemented diets. This is in accordance with the results of Ferguson et al., (1961) and Sirbu et al., (1981) who reported that hatchability related to folic acid in the egg. This disagree with the results of Roble (1993) who reported that

Fayoum J. Agric. Res. \& Dev., Vol.23, No.1, January, 2009 
PERFORMANCE OF AGED LAYING FAYOUMI HENS FED............. 7 dietary supplemental folic acid levels produced a positive linear response pattern on the transfer of folic acid in eggs, but did not result in a hatchability increase. Also, Roble (1992) found that dietary supplementation of pyridoxine above the basal diet did not result in increasing hatchability of turkey eggs.

\section{Egg Quality:}

The results obtained for egg quality in terms of egg weight $(\mathrm{EW})$, egg shape index \% (ESI), albumen (Alb.) \%, yolk (Y) \%, shell (S) \%, shell thickness (ST), albumen height $(\mathrm{AH})$ and yolk height $(\mathrm{YH})$ at 48-52, 52-56 and 56-60 weeks of age are shown in Table 4.

At all ages, hens fed pyridoxine-supplemented diet had significantly the highest EW followed by hens fed folic acid-supplemented diet and then hens fed control diet. The results also indicated that hens fed folic acid diet had significantly higher ESI \% than those fed either pyridoxine-supplemented or control diets that had similar values in this respect. The results also indicated that hens fed control diet had significantly lower Y \%, S \%, ST and YH than those fed either folic acid or pyridoxine-supplemented diets that had similar values in this respect. No significant differences were observed in both $\mathrm{Alb}$. \% and $\mathrm{AH}$ among different treatments.

The superiority of hens receiving folic acid or pyridoxine-supplemented diets in respect of shell quality observed in the current study agree with the findings of Keshavarz (2003) who found that folic acid have the potential effect to improve shell quality. Moreover, there is a little information regarding the folic acid supplementation on egg quality characters.

\section{Economical efficiency:}

Economical evaluation parameters for folic acid or pyridoxine supplementation in aged Fayoumi laying hen diets in terms of feeding cost of the experimental diets, net revenue, economical efficiency (EEf) and relative economical efficiency (REEf) of egg hatchability are listed in Table 5.

Results showed that the folic acid-supplemented diet had significantly the highest REEf \% followed by the pyridoxine-supplemented diet and then the control diet. The reason behind this is that feeding folic acid-supplemented diet caused the highest salable chick \%, but feeding pyridoxine-supplemented diet led to the medium salable chick \%. However, the lowest one was obtained in case of feeding the control diet.

From the nutritional and economical point of view, under the present experimental conditions, it is advisable to add $1 \mathrm{mg}$ folic acid or $6 \mathrm{mg}$ pyridoxine $/ \mathrm{kg}$ of aged Fayoumi laying hen diet. Such practice helps in improving laying performance, fertility, hatchability and egg quality and seems to be advantageous in decreasing chick abnormalities.

Fayoum J. Agric. Res. \& Dev., Vol.23, No.1, January, 2009 
Table 4: Egg quality traits of laying hens fed the experimental diets at 50, 54 and 58 weeks of age.

\begin{tabular}{|c|c|c|c|}
\hline \multirow{2}{*}{ Items } & \multicolumn{3}{|c|}{ Treatments } \\
\hline & Control & Folic acid & Pyridoxine \\
\hline & \multicolumn{3}{|c|}{50 wks. } \\
\hline Egg weight (EW), g & $47.61 \pm 0.16^{\mathrm{c}}$ & $50.31 \pm 0.06^{\mathrm{b}}$ & $51.13 \pm 0.12^{\mathrm{a}}$ \\
\hline Egg shape index (ESI), \% & $77.03 \pm 0.99^{b}$ & $80.69 \pm 0.93^{\mathrm{a}}$ & $77.01 \pm 0.87^{b}$ \\
\hline Albumen (Alb), \% & $56.21 \pm 0.14$ & $55.92 \pm 0.21$ & $55.85 \pm 0.19$ \\
\hline Yolk (Y), \% & $32.52 \pm 0.19^{b}$ & $33.28 \pm 0.14^{\mathrm{a}}$ & $33.41 \pm 0.11^{\mathrm{a}}$ \\
\hline Shell (S), \% & $11.01 \pm 0.08^{\mathrm{b}}$ & $11.57 \pm 0.19^{\mathrm{a}}$ & $11.58 \pm 0.11^{\mathrm{a}}$ \\
\hline Shell thickness (ST), mm & $4.07 \pm 0.06^{\mathrm{b}}$ & $4.53 \pm 0.10^{\mathrm{a}}$ & $4.46 \pm 0.17^{\mathrm{a}}$ \\
\hline Albumen height (AH) & $7.07 \pm 0.17$ & $6.36 \pm 0.11$ & $6.41 \pm 0.17$ \\
\hline \multirow[t]{2}{*}{ Yolk height (YH) } & $14.51 \pm 0.13^{\mathrm{b}}$ & $15.75 \pm 0.10^{\mathrm{a}}$ & $15.68 \pm 0.21^{\mathrm{a}}$ \\
\hline & \multicolumn{3}{|c|}{54 wks. } \\
\hline Egg weight (EW), g & $47.27 \pm 0.09^{\mathrm{b}}$ & $49.62 \pm 0.10^{\mathrm{b}}$ & $50.70 \pm .06^{\mathrm{a}}$ \\
\hline Egg shape index (ESI), \% & $77.50 \pm 0.39^{\mathrm{b}}$ & $80.99 \pm 0.73^{\mathrm{a}}$ & $77.22 \pm 0.88^{b}$ \\
\hline Albumen (Alb), \% & $57.43 \pm 0.28$ & $57.01 \pm 0.15$ & $57.13 \pm 0.30$ \\
\hline Yolk (Y), \% & $31.22 \pm 0.84^{\mathrm{b}}$ & $32.18 \pm 0.76^{\mathrm{a}}$ & $32.15 \pm 0.98^{\mathrm{a}}$ \\
\hline Shell (S), \% & $10.15 \pm 0.63^{\mathrm{b}}$ & $10.95 \pm 0.41^{\mathrm{a}}$ & $10.82 \pm 0.48^{\mathrm{a}}$ \\
\hline Shell thickness (ST), mm & $4.00 \pm 0.07^{\mathrm{b}}$ & $4.80 \pm 0.14^{\mathrm{a}}$ & $4.73 \pm 0.18^{\mathrm{a}}$ \\
\hline Albumen height (AH) & $6.85 \pm 0.10$ & $7.01 \pm 0.16$ & $6.94 \pm 0.10$ \\
\hline \multirow[t]{2}{*}{ Yolk height (YH) } & $14.07 \pm 0.70^{\mathrm{b}}$ & $14.98 \pm 0.26^{\mathrm{a}}$ & $15.06 \pm 0.10^{\mathrm{a}}$ \\
\hline & \multicolumn{3}{|c|}{58 wks. } \\
\hline Egg weight (EW), g & $48.09 \pm 0.05^{\mathrm{c}}$ & $49.00 \pm 0.06^{\mathrm{b}}$ & $49.93 \pm 0.08^{\mathrm{a}}$ \\
\hline Egg shape index (ESI), \% & $77.13 \pm 0.78^{b}$ & $79.53 \pm 0.79^{\mathrm{a}}$ & $77.10 \pm 0.91^{\mathrm{b}}$ \\
\hline Albumen (Alb), \% & $58.04 \pm 0.22$ & $57.92 \pm 0.37$ & $57.83 \pm 0.91$ \\
\hline Yolk (Y), \% & $31.15 \pm 0.31^{\mathrm{b}}$ & $32.04 \pm 0.13^{\mathrm{a}}$ & $32.01 \pm 0.73^{\mathrm{a}}$ \\
\hline Shell (S), \% & $10.12 \pm 0.15^{\mathrm{b}}$ & $11.01 \pm 0.24^{\mathrm{a}}$ & $11.02 \pm 0.28^{\mathrm{a}}$ \\
\hline Shell thickness (ST), mm & $4.03 \pm 0.07^{\mathrm{b}}$ & $4.60 \pm 0.06^{\mathrm{a}}$ & $4.64 \pm 0.14^{\mathrm{a}}$ \\
\hline Albumen height (AH) & $6.46 \pm 0.22$ & $6.73 \pm 0.19$ & $7.14 \pm 0.17$ \\
\hline \multirow[t]{2}{*}{ Yolk height (YH) } & $14.01 \pm 0.14^{\mathrm{b}}$ & $14.71 \pm 0.31^{\mathrm{a}}$ & $14.83 \pm 0.20^{\mathrm{a}}$ \\
\hline & \multicolumn{3}{|c|}{ Overall mean } \\
\hline Egg weight (EW), g & $47.66 \pm 0.07^{\mathrm{c}}$ & $49.64 \pm 0.04^{\mathrm{b}}$ & $50.59 \pm 0.06^{\mathrm{a}}$ \\
\hline Egg shape index (ESI), \% & $77.22 \pm 0.93^{\mathrm{b}}$ & $80.40 \pm 0.75^{\mathrm{a}}$ & $77.11 \pm 0.86^{\mathrm{b}}$ \\
\hline Albumen (Alb), \% & $57.23 \pm 0.32$ & $56.95 \pm 0.27$ & $56.94 \pm 0.31$ \\
\hline Yolk (Y), \% & $31.63 \pm 0.20^{\mathrm{b}}$ & $32.50 \pm 0.42^{\mathrm{a}}$ & $32.52 \pm 0.26^{\mathrm{a}}$ \\
\hline Shell (S), \% & $10.43 \pm 0.34^{\mathrm{b}}$ & $11.18 \pm 0.25^{\mathrm{a}}$ & $11.14 \pm 0.30^{\mathrm{a}}$ \\
\hline Shell thickness (ST), mm & $4.03 \pm 0.08^{b}$ & $4.64 \pm 0.06^{\mathrm{a}}$ & $4.61 \pm 0.09^{\mathrm{a}}$ \\
\hline Albumen height (AH) & $6.79 \pm 0.14$ & $6.70 \pm 0.011$ & $6.83 \pm 0.13$ \\
\hline Yolk height (YH) & $14.20 \pm 0.21^{\mathrm{b}}$ & $15.15 \pm 0.19^{\mathrm{a}}$ & $15.19 \pm 0.30^{\mathrm{a}}$ \\
\hline
\end{tabular}

$\mathrm{a}, \mathrm{b}, \mathrm{c}$ : means in the same row having different letters are significantly different at $\mathrm{p} \leq 0.05$.

Fayoum J. Agric. Res. \& Dev., Vol.23, No.1, January, 2009 
PERFORMANCE OF AGED LAYING FAYOUMI HENS FED............. 9

Table (5): Input-output analysis and economical efficiency ratio of experimental treatments during the whole period.

\begin{tabular}{|c|c|c|c|}
\hline \multirow{2}{*}{ Items } & \multicolumn{3}{|c|}{ Treatments } \\
\hline & Control & Folic acid & Pyridoxine \\
\hline FI (kg/hen) & 8.69 & 8.75 & 8.88 \\
\hline Feed price (L.E/kg) & 2.08 & 2.12 & 2.11 \\
\hline Feed cost ${ }^{*}$ (L.E/hen/period) & 18.08 & 18.55 & 18.74 \\
\hline Salable chick (No./hen/ period) & 34.04 & 37.99 & 37.29 \\
\hline Chick price $^{* *}$ (L.E/hen/period) & 51.07 & 56.99 & 55.94 \\
\hline Net revenue (L.E/hen/period) & 17.03 & 19.00 & 18.65 \\
\hline $\mathbf{E E}_{\mathbf{f}} * * *$ & 0.94 & 1.02 & 1.00 \\
\hline $\mathrm{REE}_{\mathrm{f}}{ }^{* * * * *}(\%)$ & 100 & 109 & 106 \\
\hline \multicolumn{4}{|l|}{$* \quad$ Accor } \\
\hline \multicolumn{4}{|l|}{$* *$} \\
\hline \multicolumn{4}{|c|}{$* * * \quad \mathrm{EE}_{\mathrm{f}}$ : Economical efficiency, net revenue per unit of total feed cost. } \\
\hline \multicolumn{4}{|c|}{$* * * *$ Relative economical efficiency, assuming that the control treatment $=100 \%$. } \\
\hline
\end{tabular}

\section{REFERENCES}

A.O.A.C., (1980). Official Methods of Analysis Association of Official Analytical Chemists, 15 th Edition, Washington, D.C., USA.

Bender, D.A. (1989). Vitamin $\mathrm{B}_{6}$ requirements and recommendations. Eur J Clin Nutr; 43:289-309.

Chandra, R. and L. Sudhakaran (1990). Regulation of immune responses by Vitamin $\mathrm{B}_{6}$, NY Acad Sci; 585:404-423.

Duncan, D.B., (1955). Multiple range and multiple F tests.Biometrics, 11: 1-42.

Elibol, O., S.D. Peak and J. Brake, (2002). Effect of flock age, length of egg storage, and frequency of turning during storage on hatchability of broiler hatching eggs. Poult. Sci., 81: 945-950.

Ferguson, T.M.; C.H. Whiteside; C.R. Creger; M.L. Jones; R.L. Atkinson and J. R. Couch. (1961). B-vitamin deficiency in the mature turkey hen. Poultry Sci., 40: 1153-1159.

House, J.D.; K. Braun; D.M. Balance; C.P.O., Connor and W. Guenter. (2002). The enrichment of eggs with folic acid through supplementation of the laying hen diet. Poult. Sci., 81: 1332-1337.

House. J.D.; R.L. Jacobs; L.M. Stead; M.E. Brosnan and J.T. Brosnan (1999). Regulation of homocystine metabolism. Adv. Enzyme Reg., 39: 69-91

Keshavarz, K. (2003). Effects of reducing dietary protein, methionine, choline, folic acid, and vitamin B12 during the late stages of the egg production cycle on performance and egg shell quality. Poult. Sci., 82(:1407-1414.

Kucukyilmaz, K., E. Baser, C. Erensayin, H. Orhan and E. Arat, (2001). Effect of egg weight on the hatchability, fattening performance and egg yield traits of Japanese quail. J. Central Anim. Res. Ins., 11: 6-12.

Leklem, J.E., (1999). Vitamin $B_{6}$ in: Shils ME, Olson JA, Shike M, Ross AC, ed, Moderwl Nutrition in Health and Disease, 9th ed. Baltimore: Williams and Wilkins, 413-421.

Fayoum J. Agric. Res. \& Dev., Vol.23, No.1, January, 2009 
N.R.C., (1994). Nutrient Requirements of Poultry 9th ed, National Academy of Science, National Research Council. Washinton, D.C., U.S.A.

Robel, E.J. (1992). Effect of dietary supplemental pyridoxine levels on the hatchability of turkey eggs. Poult. Sci., 71: 1733-1738.

Robel, E.J. (1993). Evaluation of egg injection of folic acid and effect of supplement folic acid on hatchability and poult weight. Poult. Sci., 72: 546-553.

Ryu, K.S.; G.M. Pesti; K.D. Roberson; HM. Jr. Edwards; and R.R. Eitenmiller (1995). The folic acid requirements of starting broiler chicks fed diets based on practical ingredients. 2. Interrelationships with dietary methionine. Poult Sci.;74:1456-1462.

Sahan, U. and A. Ipek, (2000). Effects of breeder age and incubation humidity on hatching characteristics of broiler breeder eggs. J. Poult. Res., 2: :11-14.

Seker, I.; S. Kul and M. Bayraktar (2004). Effects of parental age and hatching egg weight of Japanese quails on hatchability and chick weight. Int. J. Poult. Sci., 3: 259-265.

Shibata K.; M. Mushiage; T. Kondo; T. Tjayakawa and H. Tsuge (1995). Effects of vitamin $\mathrm{B}_{6}$ deficiency on the conversion ratio of tryptophan to niacin. Biosci Biotechnol Biochem; 59: 2060- 2063.

Sirbu, M.; C. Damian; E. Rotunjeanu and D. Turgu. (1981). Establishing the optimum level of folic acid in feeds for laying hens. Lucr. Stiint. Inst. Cerce. Pentru Nutr. Anim. 9/10: 153-160.

SPSS (1993). SPSS Statistical software Package for the Social Sciences. $2^{\text {nd }}$ ed. HA33- N48. McGrow-Hill, Inc., USA.

Steel, R. G. D. and J. H. Torrie (1980). Principles and Procedures of Statistics: A Biometrical 2 nd ed. McGraw-Hill Book Co., Inc., New York, USA.

Tona, K., F. Bamelis, W. Coucke, V. Bruggeman, and Decuypere (2001). Relationship between broiler breeder's age and egg weight loss and embryonic mortality during incubation in large-scale conditions. J. Appl. Poult Sci., 10: 221-227.

Trakatellis. A.; A. Dimitriadou and M. Trakatelli (1997). Pyridoxine deficiency: New approaches in immunosuppression and chemotherapy. Postgrad. Med J; 73: 617-622.

Fayoum J. Agric. Res. \& Dev., Vol.23, No.1, January, 2009 
PERFORMANCE OF AGED LAYING FAYOUMI HENS FED.

الآداء الإتتاجى للاجاج الفيومى البياض المتقدم فى العمر والمغذى على علائق مضاف إليها البيرودوكسين وحمض الباض الفوليك الفعر والمغ

\author{
محمد سعد بهنس \\ كلية الزراعة ـ قسم إنتاج الدواجن - جامعة الفيوم - الفيوم - مصر
}

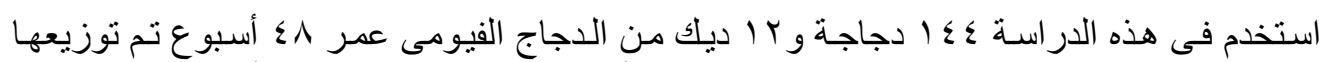

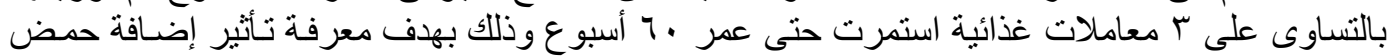

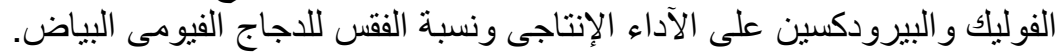

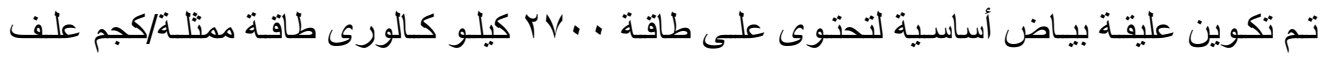

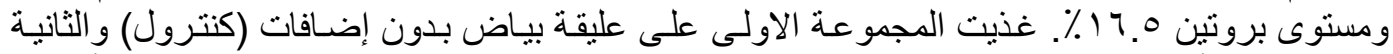

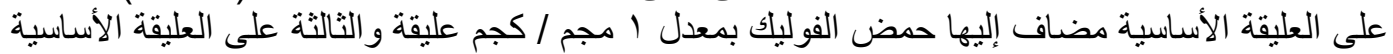

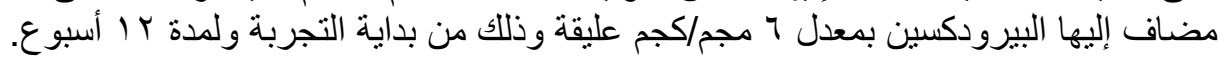

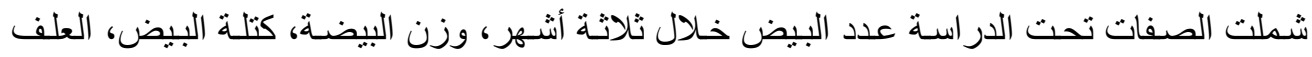

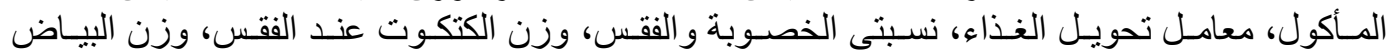

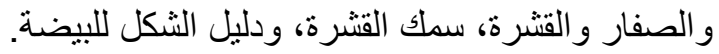
تم تحليل البيانات إحصائيا وكانت أهم النتائج المتحصل عليها كما يلى ولى الئل

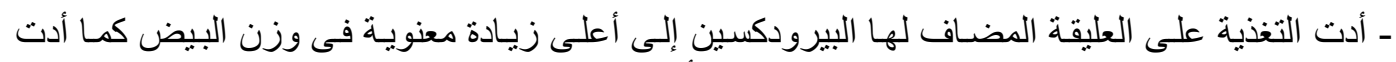

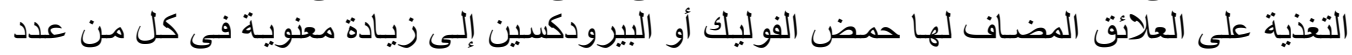
وكتلة البيض وتحسين معنوى فى معامل تحويل الغذاء مقارنة بالتغذية على عليقة الكنترول.

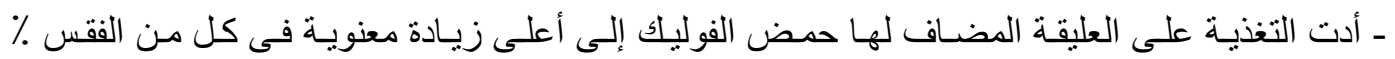

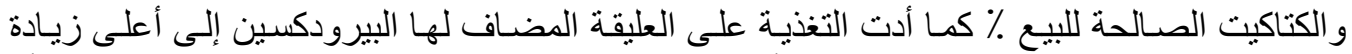

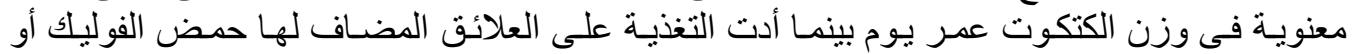

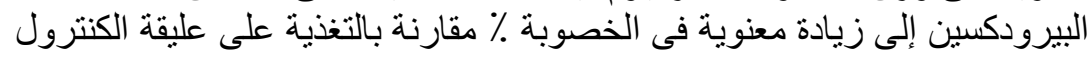

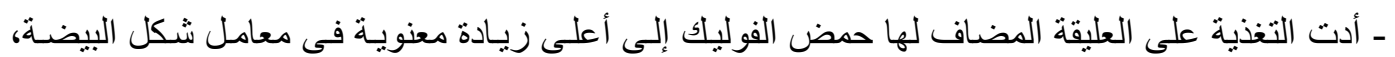

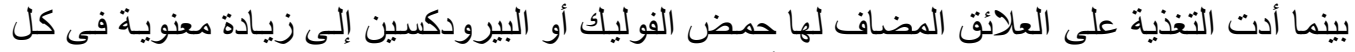

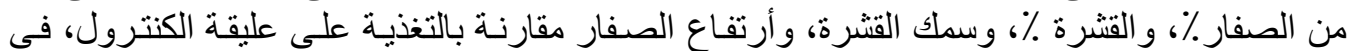

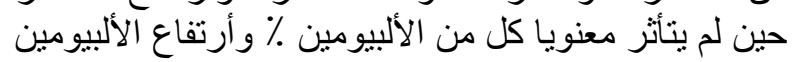

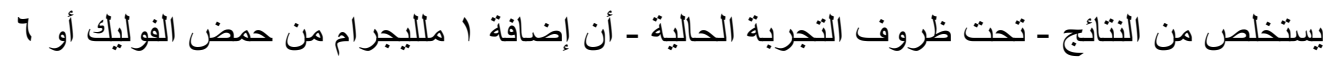

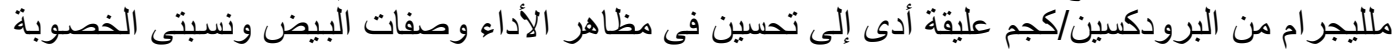

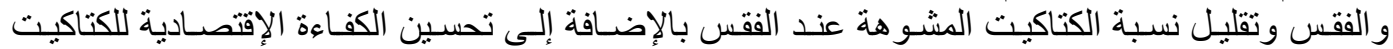

